\section{L'Actualité économique}

L'ACTUALITÉ ÉCONOMIQUE

L'Économie du Portugal - Données et problèmes de son expansion, par ALBERT PASQUIER (en collaboration avec C.-Correia Botelho). Un vol., $5^{1 / 2}$ po. $x$ 71/2, broché, 234 pages. P. PICHON et R. DURAND-AUZIAS, 20 , rue Soufflot, Paris $\left(5^{\mathrm{e}}\right)$

\title{
Jean Lotte
}

Volume 38, numéro 2, juillet-septembre 1962

URI : https://id.erudit.org/iderudit/1001792ar

DOI : https://doi.org/10.7202/1001792ar

Aller au sommaire du numéro

Éditeur(s)

HEC Montréal

ISSN

0001-771X (imprimé)

1710-3991 (numérique)

Découvrir la revue

Citer ce compte rendu

Lotte, J. (1962). Compte rendu de [L'Économie du Portugal - Données et problèmes de son expansion, par ALBERT PASQUIER (en collaboration avec C.-Correia Botelho). Un vol., 51/2 po. x 71/2, broché, 234 pages. - P. PICHON et R. DURAND-AUZIAS, 20, rue Soufflot, Paris $\left(5^{\mathrm{e}}\right)$ ]. L'Actualité économique, 38(2),

308-309. https://doi.org/10.7202/1001792ar d'utilisation que vous pouvez consulter en ligne. 
titue pas cette innovation majeure par un mariage inédit de techniques différentes que nous annoncent les éditeurs, il s'agit cependant d'un mélange assez heureux de ces techniques d'analyse pour que l'œuuve mérite d'être signalée et même recommandée comme un modèle du genre.

Pierre Harvey

\section{L'Economie du Portugal - Données et problèmes de son expansion, par ALBERT PAsQuIER (en collaboration avec C.Correia Botelho). Un vol., $51 / 2$ po. $\times 71 \frac{1}{2}$, broché, 234 pages. - P. Pichon et R. Durand-Auzias, 20, rue Soufflot, Paris $\left(5^{\circ}\right)$.}

C'est à la suite de voyages d'études que l'auteur, professeur à la Faculté de Droit et de Sciences Économiques de Caen (France), a dressé le tableau de l'économie portugaise qu'il nous présente dans ce livre. On n'y trouvera pas d'analyses de détail, mais des données générales sur un pays occidental qui reste fort peu connu. Ce travail pourrait servir d'exemple pour une approche concrète des différentes économies nationales. C'est seulement à partir de nombreuses études de ce genre que l'on devrait rechercher les principaux types de structures économiques répandus à travers le monde. Trop souvent, au contraire, on procède à l'inverse: on discourt sur ces types, que l'on suppose bien homogènes (les économies sous-développées, par exemple), sur la base de quelques caractères communs, comme le faible revenu individuel; on se situe à un niveau de généralité tel que l'on ne peut pas aboutir à des modèles descriptifs ou prospectifs véritablement utilisables dans un cas précis.

La situation du Portugal montre justement que les pays sous-développés ne forment pas une masse indistincte de nations à structiure économique identique, anonyme en quelque sorte. Chaque pays conserve ses caractéristiques propres. Dans le cas du Portugal, cette spécificité apparaît clairement à travers l'étude présentée: il s'agit d'un pays possédant les attributs du sous-développement (faible revenu, structure primaire . . .) , mais qui est loin de partir de zéro. Son originalité lui vient de ce que: 1) il possède un système institutionnel complet: organisation économique à base corporative, institutions financières, plans de développement se rapprochant du modèle français . . . 2) il ignore remarquablement les difficultés financières: aucune inflation ne se manifeste, la dette publique (intérieure et extérieure) est minime, il n'y a pas de problème de la balance des paiements, la trésorerie de l'État est très à l'aise, et l'épargne déposée dans les banques et les institutions est abondante.

Après avoir ainsi défni l'économie portugaise, l'auteur se penche sur les problèmes de sa croissance. Il constate en effet que celle-ci, en termes de produit national brut, n'a atteint que 3 p.c. par an ces dernières années, soit, en tenant compte de l'accroissement démographique, 2 p.c. per capita. Cela signifie, d'une part, que l'économie portugaise ne stagne pas: elle accomplit sa phase de démarrage; d'autre part, que ce démarrage est trop lent: les nations européennes développées réalisant des taux de croissance supérieurs, l'écart ne peut que continuer de s'accroître entre elles et le Portugal. C'est donc un problème d'accélération qui se pose à ce pays et l'auteur montre parfaitement que sa solution dépend: 1) d'une 


\section{LES LIVRES}

meilleure utilisation des ressources financières disponibles et d'une politique monétaire plus «actives. On vient d'ailleurs d'accomplir des réformes devant permettre une pleine utilisation de l'épargne existante. 2) D'une prise de conscience de la nécessité d'une meilleure productivité, ce qui suppose un esprit dynamique, encore insuffisant. Le problème n'est donc pas uniquement économique, mais aussi psychologique et sociologique pour une part égale.

La lecture de ce livre intéressera donc quiconque s'occupe du développement. On y verra comment celui-ci apparaît dans un pays européen de vieille culture. On nous permettra cependant d'exprimer une réserve sur la conclusion de l'auteur, qui oppose expansion économique et stabilité monétaire, celle-ci risquant d'étouffer celle-là au Portugal. À notre avis, il ne doit pas y avoir de conflit entre les deux, l'inflation constituant le pire ennemi du progrès. Il n'y a pas incompatibilité entre une politique monétaire active et la stabilité: le tort du Portugal réside dans une peur de l'inflation telle qu'il n'ose pas employer à fond ses ressources financières ni recourir aux techniques monétaires modernes. Entre ce ssous-emploi, de son principal atout et une politique inflationniste, il subsiste une belle marge qui devrait permettre au Portugal la croissance dans la stabilité.

Jean Lotte

La banque protestante en France, par Herbert Luthy. Un vol., $61 / 2$ po. $\times 10$, relié, 861 pages. - Ecole Pratique des Hautes Etudes, VI ${ }^{\mathrm{e}}$ section. Centre De Recherches Historiques 13, rue du Four, Paris, 1961.

L'auteur démontre dans cet ouvrage que l'opinion selon laquelle les financiers protestants français représentaient un groupe à part, est sans fondement. Herbert Luthy affirme, en effet, que tout au cours du XVIII ${ }^{e}$ siècle la société protestante était incapable de créer une unité et une homogénéité suffisante pour justifier une telle affirmation. Il considère qu'il s'agissait bien plus d'une «non-appartenance, à certaines théories du catholicisme que d'une doctrine religieuse uniforme jus. tifiant des agissements d'ordre économique.

Herbert Luthy s'attache également à décrire les effets salutaires du prêt à intérêt en tant que base de la généralisation du crédit indispensable à l'essor de l'industrie et du commerce. Selon lui le taux d'intérêt était un dénominateur permettant de calculer le risque, mal connu alors et mal évalué, mais infiniment plus important que de nos jours.

Le choix entre les deux théories, calviniste et catholique, ne s'opérait pas, en outre, en fonction d'appartenances religieuses, mais plutôt suivant les besoins propres d'une région. Les États qui ne pouvaient s'appuyer sur une richesse agricole, étaient forcés, en somme, de chercher d'autres sources de profits et d'organiser d'une façon plus efficace leur commerce et leur politique monétaire. De même que la lutte qui se livrait entre la finance et la banque n'avait pas pour origine des oppositions religieuses, mais plutôt celles de classes sociales. Les financiers servaient surtout le Roi et la Cour, tandis que les banquiers fournissaient l'argent au commerce. 